\title{
The impact of colchicine in preventing post- pericardiotomy syndrome; a double-blind clinical trial study
}

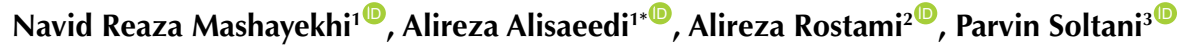 \\ 'Department of Cardiology, Arak University of Medical Sciences, Arak, Iran \\ 2Department of Cardiac Surgery, Arak University of Medical Sciences, Arak, Iran \\ ${ }^{3}$ Department of Internal Medicine, Faculty of Medicine, Arak University of Medical Sciences, Arak, Iran
}

\section{*Correspondence to Alireza Alisaeedi, Email: dr.alisaeedi@arakmu. ac.ir}

Received 11 Aug. 2019 Accepted 9 Dec. 2019 Published online 7 Jan. 2020

Keywords: Colchicine, Pericarditis, Postpericardiotomy syndrome, Open heart surgery, Cardiopulmonary bypass, Antiinflammatory

Abstract
Introduction: Post-pericardiotomy syndrome is a relatively common complication after open heart surgery. In recent
years, colchicine had a good effect on treatment of the pericarditis.
Objectives: This study aimed to study the effect of colchicine to prevent post-pericardiotomy syndrome in patients
who had open heart surgery.
Patients and Methods: This double-blind clinical trial study was conducted on 240 patients undergone open heart
surgery. Eligible patients were selected by simple sampling and randomly divided into two groups; 120 subjects in
the experimental group receive colchicine ( 1 mg for patients greater than 70 kg and 0.5 mg for patients lower than
70 kg) and 120 subjects in the control group who received the placebo. Patients were followed up regarding the
incidence of post-pericardiotomy syndrome after the surgery during hospitalization and in follow up visits in one
month and six months after hospital discharges.
Results: In total, the incidence rate of post-pericardiotomy syndrome was $33.7 \%$ (81 subjects). A significant difference
of post-pericardiotomy syndrome between two groups was seen. We found post-pericardiotomy syndrome in $12.1 \%$
(29 patients) of colchicine group versus $21.6 \%$ (52 patients) in the placebo group (chi-square test). The incidence rate
based on the Kaplan-Meier curve, in the second week to one month after the surgery, was lower in colchicine group
while in third to the sixth month it had a constant trend.
Conclusion: Colchicine is effective for prevention the post-pericardiotomy syndrome without any life-threatening
complication.
Trial registration: Registration of trial study has been approved in the Iranian Registry of Clinical Trial (identifier:
IRCT20141209020258N70; https://irct.ir/trial/28142, ethical code; IR.ARAKMU.REC92-148-11).

Citation: Mashayekhi NR, Alisaeedi A, Rostami A, Soltani P. The impact of colchicine in preventing post-pericardiotomy syndrome; a doubleblind clinical trial study. Immunopathol Persa. 2020;6(1):e11. DOI:10.15171/ ipp. 2020.11

\section{Introduction}

Post-pericardiotomy syndrome (PPS) is a relatively common complication after open heart surgery with the occurrence time of days to weeks after surgery $(1,2)$. This syndrome was first described by Janton et al as a disease including fever and pleuritic chest pain after rheumatic mitral valve repair $(3,4)$. However, it was also observed in non-rheumatic heart diseases and after heart surgery with or without cardiopulmonary bypass. PPS is currently recognized as an inflammatory complication of immune response which occurs following surgical trauma to the pericardium, pleura or both, due to the release of stimulant antigens of immune response and ultimately inflammation $(2,5)$. PPS usually occurs during the early weeks after surgery, but may also occur 6 to 12 months after surgery with less prevalence $(6,7)$. Symptoms of the disease vary depending on its severity and can be manifested as atrial fibrillation, pleural effusion, pericardial effusion, constrictive

\section{Key point}

In a double-blind clinical trial study conducted on 240 patients undergone open heart surgery, we found colchicine is effective for prevention the postpericardiotomy syndrome without any life-threatening complication.

pericarditis and cardiac tamponade. Diagnosis of this syndrome is based on the presence of at least two criteria as follows; fever of unknown origin, pleuritic chest pain, friction rub, constrictive pericarditis, cardiac tamponade, atrial fibrillation and occurrence or exacerbation of pericardial effusion and pleural effusion $(2,5,8,9)$. Very mild cases of this syndrome are usually without symptoms and are spontaneously resolved while in other cases, medical treatment is necessary. Common treatment for this complication is experimental and done using acetylsalicylic acid or non-steroidal anti-inflammatory drugs. Corticosteroids

Copyright $($ ) 2020 The Author(s); Published by Nickan Research Institute. This is an open-access article distributed under the terms of the Creative Commons Attribution License (http://creativecommons.org/licenses/by/4.0), which permits unrestricted use, distribution, and reproduction in any medium, provided the original work is properly cited. 
are prescribed in the case of contraindications or failure to respond to these treatments (10-13). In recent years, the preventive modalities for this complication are under study and evaluation. Colchicine is one of the drugs under consideration for primary prevention. This drug was initially used in the study by Finkelstein et al, to reduce PPS occurrence in 163 patients having undergone open heart surgery. It was administered since the third day after surgery for one month, and in a three-month follow-up. It was specified that colchicine outperformed in favor of reducing post-pericardiotomy syndrome (9). Likewise, Imazio et al studied the effect of colchicine on treatment and prevention of PPS and concluded that colchicine reduced the occurrence of early postoperative pericardial and pleural effusion in cardiac surgery patients. Additionally, Mack et al analyzed all the papers published during 1966-2011, regarding the effect of colchicine on treatment and prevention of PPS as a systematic review. However, no adequate data indicating colchicine as a conventional treatment for PPS prevention in patients undergone open heart surgery was detected (1). In addition, Imazio et al in a systematic review, analyzed the effect and complications of colchicine for prevention of pericarditis in 127 related published papers (a total of 795 patients), and concluded that colchicine is effective and safe for primary and secondary prevention of pericarditis (14).

\section{Objectives}

Considering the fact that studies on colchicine had contradictory results, and while no other drug has been introduced for PPS prevention and treatment, we sought to evaluate the effect of colchicine on the prevention of PPS in an intervention study with a six-month follow-up period in patients who underwent open heart surgery.

\section{Patients and Methods}

\section{Study design}

In this double-blind clinical trial study, 240 patients underwent open heart surgery (2013 to 2014) in AmirAlmomnin hospital, Arak, Iran. Participants were randomly assigned to treatment by a central computer-based, automated sequence. All participants and trial investigators were blinded to randomized treatment. Age of more than 18 years and the indication of open heart surgery were the study inclusion criteria. Patients with a contraindication of colchicine, such as severe liver disease or high levels of liver enzymes, serum creatinine of greater than $2.5 \mathrm{mg} / \mathrm{dL}$, heart failure, anemia, gastrointestinal disease, pregnancy and breastfeeding, and known sensitivity to colchicine or recent intake of this drug were excluded from the study. The case group received colchicine on the first day after surgery at the dose of $1 \mathrm{mg}$ twice per day for first day, and then received $1 \mathrm{mg}$ daily for one month in patients more than $70 \mathrm{~kg}$ and $0.5 \mathrm{mg}$ in patients less than $70 \mathrm{~kg}$. The control group received placebo. All patients were evaluated by clinical examination, ECG monitoring, chest $\mathrm{X}$-ray and echocardiography at one day after surgery at the time of discharge and one month and also six months after discharge by a cardiologist unaware of the treatment groups The flowchart of the patients included in the study is shown in Figure 1.

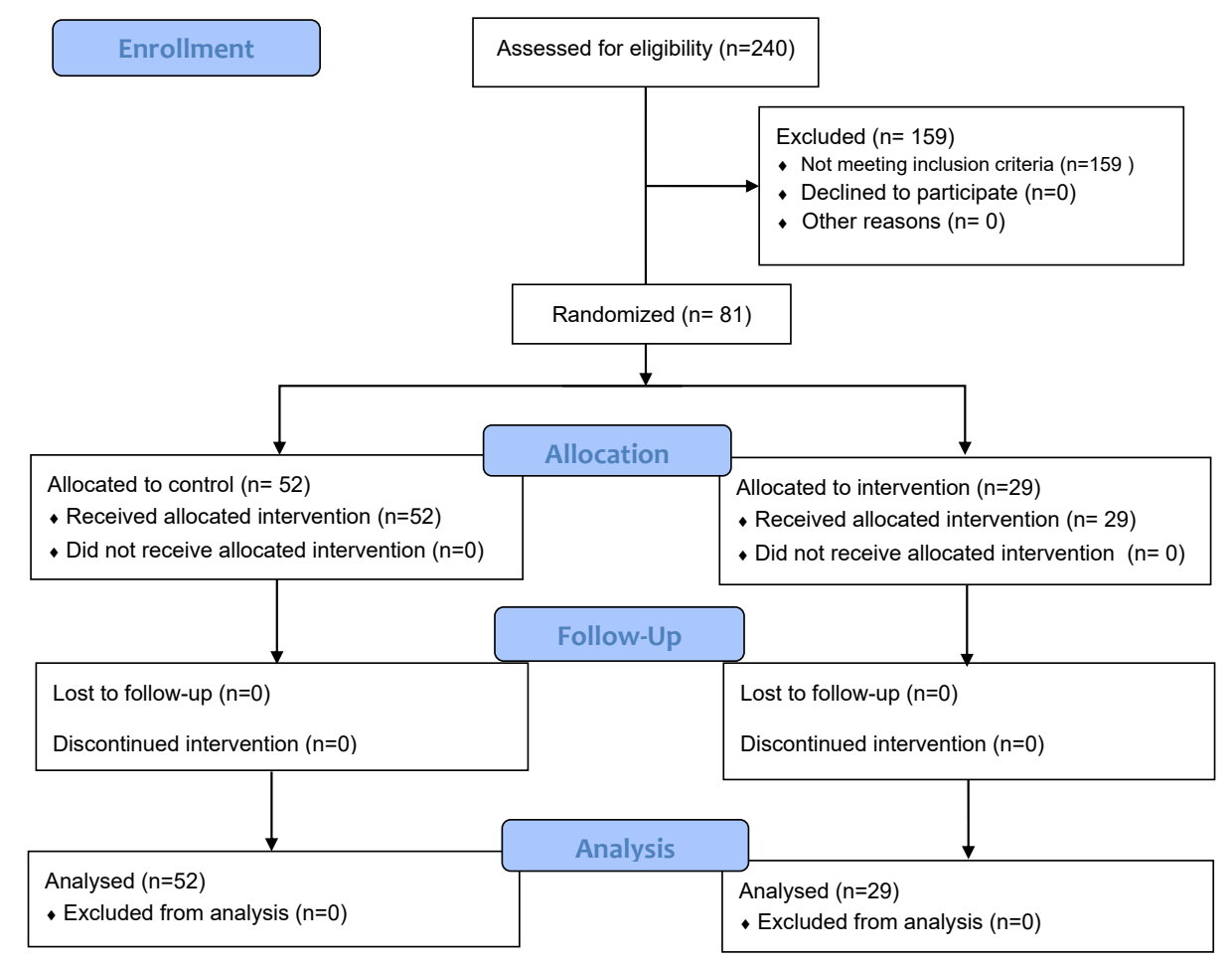

Figure 1. Flowchart of the study. 


\section{Ethical issues}

The research followed the tenets of the Declaration of Helsinki. This study was approved in the Ethics committee of Arak University of Medical Sciences (\#IR. ARAKMU.REC92-148-11). The trial study was also approved in the Iranian Clinical Trials Registry identifier: IRCT20141209020258N70; https://irct.ir/trial/28142). Accordingly, informed consent was obtained from the patients. This study is the result of the residential thesis by Navidreza Mashayekhi (Thesis\# U-925).

\section{Statistical analysis}

After collecting the necessary data, they were analyzed using SPSS 18 software. Accordingly, t-test or its nonparametric equivalent (Chi-square test) and observation iteration test were used. The Fisher's exact test was applied, wherever appropriate. All $P$ values were 2-tailed, and the values of less than or equal to 0.05 were considered statistically significant.

Results

This study showed that the two groups had identical

Table 1. Comparison of quantitative variables at the beginning of research in two groups

\begin{tabular}{llll}
\hline Variable & Colchicine $(\mathbf{n = 2 9 )}$ & Placebo $(\mathbf{n = 5 2 )}$ & $\boldsymbol{P}$ value* \\
\hline Age $(\mathrm{y})$ & $64.1 \pm 9.2$ & $59.2 \pm 10.1$ & 0.08 \\
BMI $\left(\mathrm{kg} / \mathrm{m}^{2}\right)$ & $27.6 \pm 2.1$ & $26.3 \pm 1.9$ & 0.4 \\
Pump time $(\mathrm{min})$ & $88.5 \pm 22.5$ & $86.3 \pm 26.1$ & 0.6 \\
Cross clamp time $(\mathrm{min})$ & $55.5 \pm 19.2$ & $52.6 \pm 16.3$ & 0.14 \\
Serum creatinine $(\mathrm{mg} / \mathrm{dL})$ & $1.3 \pm 0.3$ & $1.1 \pm 0.2$ & 0.3 \\
\hline
\end{tabular}

BMI; body mass index.

Data are presented as Mean \pm SD. ${ }^{*} t$ test independent. distribution at the beginning of the study in terms of some confounding and intervening variables based on independent group's $t$ test (Table 1).

Additionally, comparison of frequency distribution of qualitative variables such as gender ratio, patient functional class according to NYHA classification and some other qualitative variables at the beginning of research (based on chi-square test) are presented in Table 2.

The occurrence of PPS, based on the presence of at least two criteria of fever of unknown origin, pleuritic chest pain, friction rub, constrictive pericarditis, cardiac tamponade, atrial fibrillation, and occurrence or exacerbation of pericardial effusion and pleural effusion, was 12.1 percent $(\mathrm{n}=29)$ in colchicine group versus 21.6 percent $(\mathrm{n}=$ $52)$ in placebo group $(P<0.05)$. Occurrence of primary and secondary consequences of PPS was studied during hospitalization, one and six months later. The results are given in Table 3.

Likewise, the incidence of PPS showed no significant difference regarding the time immediately and one week after surgery in two groups ( $P>0.05$; Kaplan-Meier curve), however in the second week up to one month later, it was significantly lower in the colchicine group $(P<0.05)$ while it was stable in both groups in the third to the sixth months of follow up (Figure 2).

The most prevalent complication of colchicine was gastrointestinal complications. The frequency distribution of other complications is illustrated in Table 4.

\section{Discussion}

In this double-blind clinical trial study, we sought to determine the effect of colchicine on the prevention of post-pericardiotomy syndrome. We found both groups

Table 2. Comparison of frequency distribution of qualitative variables at the beginning of research

\begin{tabular}{|c|c|c|c|c|}
\hline \multirow{2}{*}{ Variable } & & \multicolumn{2}{|c|}{ Group } & \multirow{2}{*}{$P$ value* } \\
\hline & & Colchicine $(n=29)$ & Placebo $(n=52)$ & \\
\hline \multirow{2}{*}{ Gender } & Male & $12(42.3)$ & $22(43.1)$ & \multirow{2}{*}{0.34} \\
\hline & Female & $17(57.7)$ & $30(56.9)$ & \\
\hline \multirow{4}{*}{ Medical history } & Hypertension & $20(69.4)$ & $35(67.2)$ & \multirow{4}{*}{0.58} \\
\hline & Diabetes & $7(25)$ & $12(22.7)$ & \\
\hline & Smoking & $3(11.9)$ & $8(15)$ & \\
\hline & Previous MI & $6(21.6)$ & $10(19.3)$ & \\
\hline \multirow{4}{*}{ Drugs } & $\beta$-Blockers & $17(57.7)$ & $29(55.8)$ & \multirow{4}{*}{0.47} \\
\hline & Amiodarone & $3(10.2)$ & $4(7.9)$ & \\
\hline & ACE Inhibitors/ARBs & $14(47.9)$ & $29(55.6)$ & \\
\hline & Other drugs & $12(39.8)$ & $24(45.2)$ & \\
\hline \multirow{3}{*}{ NYHA functional class } & II & $20(68.4)$ & $29(63.5)$ & \multirow{3}{*}{0.59} \\
\hline & III & $6(22.3)$ & $12(23.6)$ & \\
\hline & IV & $3(9.3)$ & $7(12.9)$ & \\
\hline \multirow{3}{*}{ Type of surgery } & CABG & $27(93.2)$ & $49(96.2)$ & \multirow{3}{*}{0.9} \\
\hline & Valvular diseases & $1(3.4)$ & $1(1.9)$ & \\
\hline & Aortic disease & $1(3.4)$ & $1(1.9)$ & \\
\hline \multirow{2}{*}{ Method of surgery } & On pump & $29(100)$ & $52(100)$ & \multirow[b]{2}{*}{0.9} \\
\hline & Off pump & $0(0)$ & $0(0)$ & \\
\hline
\end{tabular}

Data are presented as No. (\%).

*Chi-square. 
Table 3. Occurrence of consequences and diagnostic criteria of PPS within six months

\begin{tabular}{|c|c|c|c|c|}
\hline \multirow{2}{*}{ Variable } & & \multicolumn{2}{|c|}{ Group } & \multirow{2}{*}{$P$ value* } \\
\hline & & Colchicine $(n=29)$ & Placebo $(n=52)$ & \\
\hline \multirow{8}{*}{ Primary outcomes } & Fever of unknown origin for more than a week & $1(3.3 \%)$ & $2(3.9 \%)$ & 0.38 \\
\hline & Pleuritic chest pain & $1(3.9 \%)$ & $7(12.8 \%)$ & 0.002 \\
\hline & Friction rub & $1(2.7 \%)$ & $4(8.3 \%)$ & 0.001 \\
\hline & Pleural effusion & $4(12.2 \%)$ & $13(25.6 \%)$ & 0.001 \\
\hline & pericardial effusion & $4(12.8 \%)$ & $12(22.8 \%)$ & 0.002 \\
\hline & Atrial fibrillation & $7(23.9 \%)$ & $13(25.7 \%)$ & 0.059 \\
\hline & Cardiac tamponade & $1(0.06 \%)$ & $1(1.7 \%)$ & 0.237 \\
\hline & Constrictive pericarditis & $0(0 \%)$ & $0(0 \%)$ & 0.9 \\
\hline \multirow{3}{*}{ Secondary outcomes } & Recurrent pleural or pericardial effusion & $1(0.9 \%)$ & $1(2.1 \%)$ & 0.001 \\
\hline & Readmission because of PPS & $2(2.2 \%)$ & $3(6.2 \%)$ & 0.003 \\
\hline & Mortality & $1(1.3 \%)$ & $1(1.9 \%)$ & 0.592 \\
\hline
\end{tabular}

Data are presented as No. (\%).

*Chi- square.

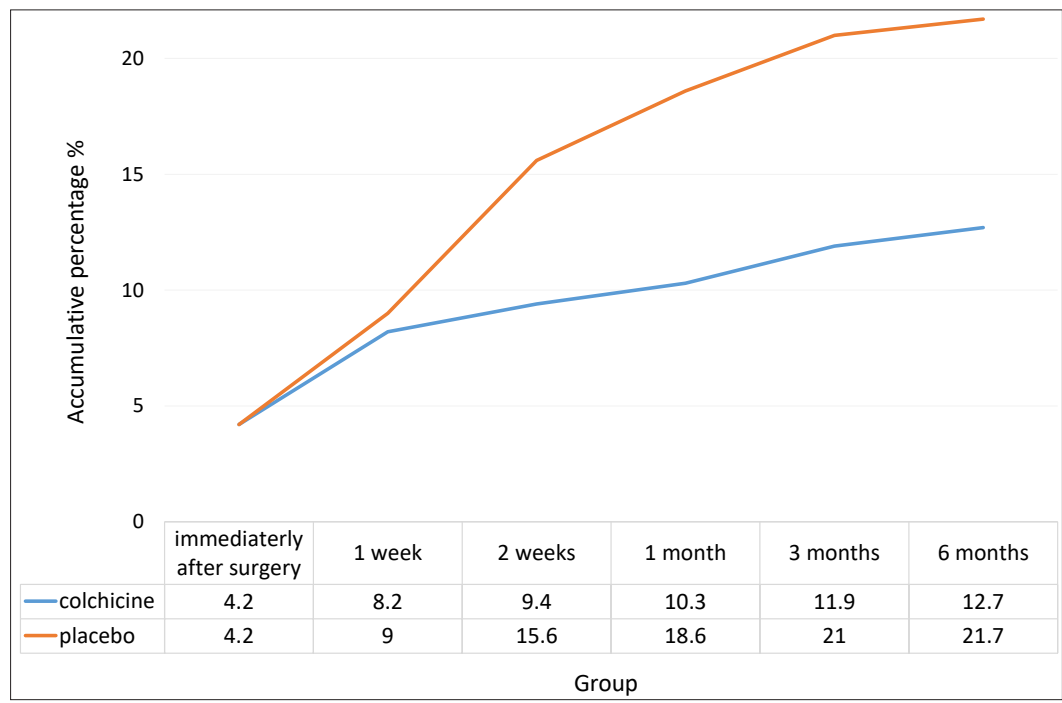

Figure 2. Accumulative percentage of post-pericardiotomy occurrence in terms of time.

had identical distribution at the beginning of the study regarding the distribution frequency of qualitative and quantitative variables, and homogeneity of both groups regarding possible confounding variables. Functional level of patients before surgery was generally at class 2 of New York classification, and the most prevalent reason for surgery was coronary artery bypass graft (CABG). The disease was a little more incident in women, but the difference was not significant. Overall, PPS occurrence in all the subjects was $33.7 \%(\mathrm{n}=81), 12.1 \%(\mathrm{n}=29)$ in the colchicine group and 21.6 percent $(n=52)$ in placebo group.

In the six-month follow-up, fever, constrictive pericarditis, cardiac tamponade, and atrial fibrillation had identical occurrences in both groups, but pleuritic chest pain and friction rub occurred less in the colchicine group. Regarding the secondary outcomes, recurrence and re-hospitalization were less in the colchicine group, but showed no change in mortality in both groups.

PPS occurrence in terms of time, immediately after surgery and one week after surgery showed no significant difference in both groups. However, in the second week up to one month later, it was lower in the colchicine group, while a stable and similar trend was observed in both groups during the third month to the sixth month.

The most prevalent complication of colchicine was gastrointestinal disorder. Using this drug with the dosage used in this work showed no life-threatening liver or kidney complications.

The results of this study are similar to those of most studies (15-22) suggesting the efficacy and safety of colchicine in preventing PPS. In a few studies, there are also different and contradictory results (23).

The potential efficacy of colchicine in preventing PPS was first introduced in 2002 (9). In this study, colchicine with a dose of $1.5 \mathrm{mg}$ /day was compared to placebo, which began on the third day after surgery on 163 patients undergoing heart surgery since 52 patients $(31.9 \%)$ were excluded from the study at the beginning of the trial due to postoperative complications or non-cooperation or gastroenteritis. During the next three months, 19 patients (17.1\%) conflicted by PPS. From 19 patients, 5 were in the 
Table 4. Side effects of colchicine in both groups

\begin{tabular}{lccl}
\hline \multirow{2}{*}{ Complications } & \multicolumn{2}{c}{ Group } & \multirow{2}{*}{$\boldsymbol{P}$ value } \\
\cline { 2 - 3 } & Colchicine & Placebo & \\
\hline Gastrointestinal* & $5(16.9)$ & $3(5.1)$ & 0.001 \\
\hline Liver ** & $1(1.7)$ & $1(0.9)$ & 0.392 \\
Renal *** & $1(2.2)$ & $2(3.8)$ & 0.51 \\
\hline Other & $6(19.7)$ & $5(10.1)$ & 0.001 \\
Life threatening complications & $0(0)$ & $0(0)$ & 0.9 \\
\hline $\begin{array}{l}\text { Data are presented as } \mathrm{n}(\%) . \\
\text { * Incidence of diarrhea, nausea, vomiting and abdominal pain; }\end{array}$ \\
** Any abnormal increase in ALT and bilirubin higher than normal range; \\
*** Any increase beyond normal range in serum creatinine or abnormal GFR \\
reduction.
\end{tabular}

colchicine group and 14 ones were in the placebo group. Results showed that colchicine is probably effective in PPS prevention, which is consistent with the findings in the current study.

No specific drug has been introduced currently for the treatment and prevention of PPS $(24,25)$. However, according to European guidelines on diagnosis and treatment of pericarditis (2004), colchicine is the first medical step for chronic pericarditis (class I indication) and it is a relatively common modality for treating acute pericarditis (class IIa indication) (26). Similarly, in the COPP study (6), colchicine was also introduced as an effective and safe drug for prevention and treatment of PPS. Our study showed that most complications of PPS (85\%) occur in the first month after surgery. Therefore, using colchicine for 4 weeks does not provide serious complications; however the risk of gastrointestinal complications and especially gastroenteritis is high. Diarrhea is a common complication of this drug (10\% of the patients) that can be controlled and reduced by adjusting the dose and choosing the right patient.

In the COOP2 study (27), colchicine had an acceptable preventive effect on this syndrome. However, similar to the results of our study, no reduction in the incidence of atrial fibrillation, pericardial effusion and pleural effusion was seen. The probability of gastrointestinal complications was also high in this study, while 20 percent of the patients were not able to continue the study,

The exact mechanism of colchicine subsiding of PPS is not still properly clear. However, its effects are mostly ascribed to inhibition and destruction of microtubules (28). Colchicine contributes to formation process of microtubules, and ultimately leads to establishment of colchicine - tubulin complex by formation of b-tubulin. This occurs in the mitotic phase or the interphase phase. Thus, colchicine leads to inhibition of intracellular granules and the release of various substances (29). By this mechanism, colchicine can inhibit various functions of leukocytes and finally anti-inflammatory function. In addition, colchicine is able to accumulate in leukocyte, thereby its concentration in leukocytes is 16 times higher than plasma levels. This mechanism influences its medical effects.
Various and different treatments have been utilized for PPS prevention including aspirin, corticosteroids, and colchicine. In a meta-analysis on 894 patients, colchicine was reported as an effective drug in preventing from this syndrome $(25,30,31)$, while aspirin and corticosteroids have milder effects. In a single-center study with a sample size of 822 subjects treated with dexamethasone or placebo, it was found that corticosteroids had a little preventive effect on the appearance of PPS (32). However, administration of colchicine has been shown to have preventive effects on the simultaneous increase in C-reactive protein and the development of anti-inflammatory properties. This antiinflammatory mechanism justifies that it should not be expected that colchicine influences all PPS complications such as atrial fibrillation or fluid effusion (8). On the other hand, this overall anti-inflammatory mechanism of colchicine justifies the occurrence of this syndrome at the first week after surgery in the current study, and later, the syndrome probability reduces with the emergence of antiinflammatory effects. The occurrence of complications and non-cooperation of patients are the main obstacles to lack of early initiation of colchicine. However, considering the emergence of anti-inflammatory effects of this drug within two or three days, it is logical to start the drug up to two days before surgery with an adjusted dosage (6).

Postoperative atrial fibrillation is one of the most common complications after heart surgery and leads to increased morbidity, prolonged hospitalization and medical expenses (8-33). Pathophysiology of this fibrillation is also numerous and includes cases such as pericardial inflammation, disturbance of postoperative autoimmune balance and fluid shift (34-36). Microtubules play a significant role in the intracellular transmissions since colchicine is also effective in controlling this activity. On the other hand, colchicine influences the activity of neutrophils, and migration to the affected area (36). Therefore, it is expected that use of this drug is also effective in preventing fibrillation too. Additionally, other mechanisms may be involved in the development of this complication, which require separate recognition and intervention.

\section{Conclusion}

Administration of colchicine is effective in prevention of most complications and symptoms of post-pericardiotomy syndrome without any life-threatening complications. Of course, chance of patient non-compliance is high particularly due to gastrointestinal complications that can be controlled by adjusting the dose. In addition, the relatively high incidence of PPS at the first week after surgery in the placebo group may denote the antiinflammatory mechanism of colchicine in preventing this syndrome and early prescription of this drug (for example, one week before elective surgeries) may be effective to prevent PPS. 


\section{Limitations of the study}

According to low number of valve replacement surgery in this study, it was not possible to differentiate the incidence of PPS in subgroups of CABG against valve replacement. Also, due to the fact that the follow up period in this study was six months, it was not possible to investigate the incidence of late PPS complications. Likewise, multicentered studies, studies with larger sample size and longer follow-up, or studies on specific groups and surgeries and conducting further meta-analysis are necessary for identifying the preventive effects of colchicine on PPS complications and symptoms.

\section{Acknowledgments}

We sincerely appreciate the collaboration of patients and their families for their participation in the research.

\section{Authors' contribution}

All authors passed four criteria for authorship contribution based on recommendations of the International Committee of Medical Journal Editors. NRM and AA designed the protocol of study. AS developed the protocol and performed it. Critical revision of the manuscript for important intellectual content was performed by AR, PS and NM. Analysis of data was performed by AR. All authors read and approved the final paper.

\section{Conflicts of interest}

The authors declare that they do not have any conflict of interest.

\section{Ethical considerations}

Ethical issues (including plagiarism, data fabrication, double publication) have been completely observed by the authors.

\section{Funding/Support}

None.

\section{References}

1. Mack DR, Cahoon WD, Lowe DK. Colchicine for the primary prevention of the post-pericardiotomy syndrome. Ann Pharmacother. 2011;45:803-6. doi: 10.1345/aph.1Q112.

2. Imazio M, Brucate $\mathrm{A}$, Adler $\mathrm{Y}$. Is possible to prevent the post pericardiotomy syndrome? Int J Cardiol. 2012;159:1-4. doi: 10.1016/j.ijcard.2012.01.034

3. Janton $\mathrm{OH}$, Golver RP, O'Neill TJ, Gregory JE, Froid GF. Results of the surgical treatment fot mitral stenosis: analysis of 100 cases. Circulation. 1952;6:321-33.

4. Soloff LA, Zatuchui J, Janton OH, O'Neill TJ, Glover RP. Reactivation of rheumatic fever following mitral commissurotomy. Circulation. 1953;8:481-93.

5. Imazio M, Brucato A, Rovere ME, Gandino A, Cemin R, Ferrua $S$, et al. Colchicine prevents early postoperative pericardial and pleural effusions. Am Heart J. 2011;162:527-32. doi: 10.1016/j.ahj.2011.05.017.

6. Imazio $M$, Trinchero $R$, Brucato $A$, Rovere $M E$, Gandino $A$, Cemin $R$, et al. Colchicine for the prevention of the post-pericardiotomy syndrome (COPPS): a multicenter, randomized, double-blind, placebo-controlled trial. Eur Heart J. 2010;31:2749-54. doi: 10.1093/eurheartj/ehq319.

7. Wessman DE, Stafford CM. The postcardiac injury syndrome: case report and review of the literature. South Med J. 2006;99:309-14.

8. Spodick DH. Traumatic pericardial disease: accidental, criminal, surgical and biological trauma. In: Spodick DH, ed., the Pericardium: A Comprehensive Textbook. New York: Dekker;1997:368-432.

9. Finkelstein Y, Shemesh J, Mahlab K, Abramov D, Bar-ElY, Sagie A, et al. Colchicine for the prevention of post-pericardiotomy syndrome. Herz. 2002;27:791-4.

10. Gill PJ, Forbes K, Coe JY. The effect of short-term prophylactic acetylsalicylic acid on the incidence of post-pericardiotomy syndrome after surgical closure of atrial septal defects. Pedatr Cardiol. 2009;30:1061-7. doi: 10.1007/s00246-009-9495-1.

11. Engle MA, Zabriskie JB, Senterfit LB, Gay Jr WA, O'Loughlin Jr JE, Ehlers $\mathrm{KH}$. Viral illness and the post-pericardiotomy syndrome: a prospective study in children. Circulation. 1980; 62:1151-8.

12. Stelzner TJ, King Jr TE, Antony VB, Sahn SA. The pleuropulmonary manifestations of the postcardiac injury syndrome. Chest. 1983;84:383-7.

13. Caforio AL, Brucato A, Doria A, Brambilla G, Angelini A, Ghirardello A, et al. Anti-heart and anti-intercalated disk autoantibodies: evidence for autoimmunity in idiopathic recurrent acute pericarditis. Heart. 2010;96:779-84. doi: 10.1136/hrt.2009.187138.

14. Imazio M, Brucato A. Forno D, Ferro S, Belli R, Trinchero R, et al. Efficacy and safety of colchicine for pericarditis prevention. Systematic review and meta-analysis. Heart. 2010;98:107882.

15. Imazio M, Bobbio M, Cecchi E, Demarie D, Demichelis B, Pomari $\mathrm{F}$, et al. Colchicine in addition to conventional therapy for acute pericarditis. Circulation. 2005;112:2012-16.

16. De La Serna AR, Soldevila JG, Claramunt VM, De Luna AB. Colchicine for recurrent pericarditis. Lancet. 1987; 2:1517.

17. Guindo J, Rodriguez de la Serna A, Ramio J, de Miguel Diaz MA, Subirana MT, Perez Ayuso MJ, et al. Recurrent pericarditis: relief with colchicine. Circulation. 1990;82:1117-20.

18. Adler Y, Zandman-Goddard G, Ravid M, Avidan B, Zemer $D$, Ehrenfeld $M$, et al. Usefulness of colchicine in preventing recurrences of pericarditis. Am J Cardiol. 1994; 73:916-7.

19. Brucato A, Brambilla G, Adler Y, Spodick DH, Canesi B. Therapy for recurrent acute pericarditis: a rheumatologically solution? Clin Exp Rheumatol. 2006;24:45-50.

20. Guindo J, Adler Y, Spodick DH, De La Serna AR, Shoenfeld Y, Daniel-Riesco C, et al. Colchicine for recurrent pericarditis: 51 patients followed up for 10 years. Circulation. 1997;96:1 29

21. Artom G, Koren-Morag N, Spodick DH, Brucato A, Guindo J, Bayes-de-Luna A, et al. Pretreatment with corticosteroids attenuates the efficacy of colchicine in preventing recurrent pericarditis: a multi-center all-case analysis. Eur Heart J. 2005; 26:723-7. doi:10.1093/eurheartj/ehi197.

22. Imazio $M$, Demichelis B, Parrini I, Cecchi E, Demarie D, Ghisio A, et al. Management, risk factors, and outcomes in recurrent pericarditis. Am J Cardiol. 2005;96:736-9.

23. Mack DR, Cahoon Jr WD, Lowe DK. Colchicine for the primary prevention of the post-pericardiotomy syndrome. Ann Pharmacother. 2011;45:803-6.

24. Gill PJ, Forbes K, Coe JY. The effect of short-term prophylactic acetylsalicylic action the incidence of post-pericardiotomy syndrome after surgical closure of atrial septal defects. Pediatr Cardiol. 2009;30:1061-7.

25. Mott AR, Fraser CD, Kusnoor AV, Giesecke NM, Reul GJ, Drescher KL, et al. The effect of short-term prophylactic methylprednisolone on the incidence and severity of postpericardiotomy syndrome in children undergoing cardiac surgery with cardiopulmonary bypass. J Am CollCardiol. 2001; 37:1700-6. doi:10.1016/s0735-1097(01)01223-2.

26. Maisch B, Seferovic PM, Ristic AD, Erbel R, Rienmuller R, Adler $\mathrm{Y}$, et al. Task Force on the Diagnosis, Management of 
Pericardial Diseases of the European Society of Cardiology. Guidelines on the Diagnosis and Management of Pericardial Diseases. Eur Heart J. 2004;25:587-610.

27. Imazio M, Brucato A, Ferrazzi P. Colchicine for Prevention of Post-pericardiotomy Syndrome and Postoperative Atrial Fibrillation the COPPS-2 Randomized Clinical Trial. JAMA. 2014;312:1016-23. doi:10.1001/jama.2014.11026.

28. Imazio M, Brucato A, Trinchero R, Spodick DH, Adler Y. Colchicine for pericarditis: hype or hope? Eur Heart J. 2009; 30:532-9.

29. Adler Y, Finkelstein Y, Guindo J, Rodriguez de la Serna A, ShoenfeldY, Bayes-Genis A, et al. Colchicine treatment for recurrent pericarditis: a decade of experience. Circulation. 1998; 97:2183-5.

30. Imazio $M$, Trinchero $R$, Brucato $A$, Rovere ME, Gandino A, Cemin R, et al. COPPS Investigators. Colchicine for the Prevention of the Post-pericardiotomy Syndrome (COPPS): a multicenter, randomized, double-blind, placebo-controlled trial. Eur Heart J. 2010;31:2749-54. doi: 10.1093/eurheartj/ ehq319.

31. Horneffer PJ, Miller RH, Pearson TA, Rykiel MF, Reitz BA, Gardner TJ. The effective treatment of post-pericardiotomy syndrome after cardiac operations: a randomized placebo- controlled trial. J Thorac Cardiovasc Surg. 1990;100:292-296.

32. Imazio M, Brucato A, Markel G, Cemin R, Trinchero R, Spodick $\mathrm{DH}$, et al. Meta-analysis of randomized trials focusing on prevention of the post-pericardiotomy syndrome. Am J Cardiol. 2011;108:575-579.

33. Mitchell LB, CCS Atrial Fibrillation Guidelines Committee. Canadian Cardiovascular Society atrial fibrillation guidelines 2010: prevention and treatment of atrial fibrillation following cardiac surgery. Can J Cardiol. 2011;27:91-7. doi: 10.1016/j. cjca.2010.11.005

34. Abdelhadi RH, Gurm HS, Van Wagoner DR, Chung MK. Relation of an exaggerated rise in white blood cells after coronary bypass or cardiac valve -surgery to development of atrial fibrillation postoperatively. Am J Cardiol. 2004;93:11768.

35. Ishii Y, Schuessler RB, Gaynor SL, Yamada K, Fu AS, Boineau JP, et al. Inflammation of atrium after cardiac surgery is associated with inhomogeneity of atrial conduction and atrial fibrillation. Circulation. 2005;111:2881-8.

36. VanWagoner DR. Colchicine for the prevention of postoperative atrial fibrillation: a new indication for a very old drug? Circulation. 2011;124:2281-2282. doi: 10.1161/ CIRCULATIONAHA.111.057075. 